\title{
LightSpeed: A Compact, High-Speed Optical-Link-Based 3D Optoacoustic Imager
}

Ozsoy, Cagla ; Cossettini, Andrea ; Ozbek, Ali ; Vostrikov, Sergei ; Hager, Pascal ; Dean-Ben, Xose Luis ; Benini, Luca ; Razansky, Daniel

\begin{abstract}
Wide-scale adoption of optoacoustic imaging in biology and medicine critically depends on availability of affordable scanners combining ease of operation with optimal imaging performance. Here we introduce LightSpeed: a low-cost real-time volumetric handheld optoacoustic imager based on a new compact software-defined ultrasound digital acquisition platform and a pulsed laser diode. It supports the simultaneous signal acquisition from up to 192 ultrasound channels and provides a hig-bandwidth direct optical link (2x 100G Ethernet) to the host-PC for ultra-high frame rate image acquisitions. We demonstrate use of the system for ultrafast $(500 \mathrm{~Hz}) 3 \mathrm{D}$ human angiography with a rapidly moving handheld probe. LightSpeed attained image quality comparable with a conventional optoacoustic imaging systems employing bulky acquisition electronics and a Q-switched pulsed laser. Our results thus pave the way towards a new generation of compact, affordable and high-performance optoacoustic scanners.
\end{abstract}

DOI: https://doi.org/10.1109/tmi.2021.3070833

Posted at the Zurich Open Repository and Archive, University of Zurich

ZORA URL: https://doi.org/10.5167/uzh-211691

Journal Article

Accepted Version

Originally published at:

Ozsoy, Cagla; Cossettini, Andrea; Ozbek, Ali; Vostrikov, Sergei; Hager, Pascal; Dean-Ben, Xose Luis; Benini, Luca; Razansky, Daniel (2021). LightSpeed: A Compact, High-Speed Optical-Link-Based 3D Optoacoustic Imager. IEEE Transactions on Medical Imaging, 40(8):2023-2029.

DOI: https://doi.org/10.1109/tmi.2021.3070833 


\title{
LightSpeed: a compact, high-speed optical-link- based 3D optoacoustic imager
}

\author{
Çağla Özsoy, Andrea Cossettini, Ali Özbek, Sergei Vostrikov, Pascal Hager, Xosé Luís Deán-Ben, \\ Luca Benini, Daniel Razansky
}

\begin{abstract}
Wide-scale adoption of optoacoustic imaging in biology and medicine critically depends on availability of affordable scanners combining ease of operation with optimal imaging performance. Here we introduce LightSpeed: a low-cost real-time volumetric handheld optoacoustic imager based on a new compact softwaredefined ultrasound digital acquisition platform and a pulsed laser diode. It supports the simultaneous signal acquisition from up to 192 ultrasound channels and provides a higbandwidth direct optical link (2x 100G Ethernet) to the hostPC for ultra-high frame rate image acquisitions. We demonstrate use of the system for ultrafast $(500 \mathrm{~Hz})$ 3D human angiography with a rapidly moving handheld probe. LightSpeed attained image quality comparable with a conventional optoacoustic imaging systems employing bulky acquisition electronics and a Q-switched pulsed laser. Our results thus pave the way towards a new generation of compact, affordable and high-performance optoacoustic scanners.
\end{abstract}

Index Terms-3D imaging, data acquisition, high-speed imaging, optoacoustic, ultrasound, photoacoustic.

\section{INTRODUCTION}

$\mathrm{O}$ PTOACOUSTIC tomography (OAT) is rapidly growing to become an essential tool in many biomedical and clinical disciplines, including cancer research, dermatology, neuroscience, cardiovascular biology, to name a few examples [1]- [2] [3] [4] [5] [6] [7] [8] [9]. Dedicated handheld OAT scanners have been used in pilot clinical trials offering promising prospects for translating the technology toward precision diagnostics [10]- [11] [12]. Being a relatively recent development in the pantheon of biomedical imaging modalities, OAT commonly employs suboptimal hardware and software components. In particular, signal digitization generally relies on expensive and bulky digital acquisition systems [11], [12], hampering realization of affordable and compact systems for clinical applications. Mainstream pulse-echo ultrasound (US) systems are undergoing major technical advancements [13]. Particularly, owing to analog-to-digital conversion and partial processing embedded on the US probe, the need for bulky cables and hardware can be avoided, facilitating development of new generation digital US imaging systems with reduced size, complexity, and cost [14], [15]. OAT similarly relies on the acquisition of time-resolved (radio-frequency) US signals and hence it can greatly benefit from the development of highly integrated electronics.

Fundamental differences between US and OAT imaging must be taken into account when designing optimal signal acquisition interfaces. Specifically, OAT operates in a pure detection mode by recording very weak US signals induced by absorption of pulsed light in tissues. Those usually have orders of magnitude lower acoustic pressures compared to US echoes, i.e. often reaching sub-Pascal level. Furthermore, no compounding is involved in the formation of OAT image, thus it only takes a single nanosecond-duration laser pulse to generate a full tomographic dataset, both in 2D [16], [17] and 3D [18], [19]. In theory, this enables effectively "freezing" the tissue motion and imaging at frame rates ultimately limited only by the unidirectional propagation of ultrasound through the region of interest. In practice, the temporal resolution of OAT is effectively constrained by the data throughput capacity of imaging systems. State-of-the-art 3D imagers have been shown to attain volumetric frame rates of the order of $100 \mathrm{~Hz}$ when operating with simultaneous signal acquisition from 512 channels, thus enabling the visualization of fast biological processes, such as cardiovascular dynamics or neuronal activity [20], [21]. The imaging speed was chiefly hampered by the limited capacity of the 1 Gbps Ethernet link and the imaging

X. L. D. B. acknowledges support from the Werner und Hedy Berger-Janser Stiftung (Application No 08/2019) and the Helmut Horten Stiftung (Project Deep Skin). D. R. acknowledges support from the European Research Council under grant agreement ERC-CoG_2015_682379.

Ç. Ö. is with the Institute for Biomedical Engineering and Institute of Pharmacology and Toxicology, University of Zurich and ETH Zurich, Zurich, 8092 Switzerland (e-mail: oezsoyc@ethz.ch).

A. C. is with the Integrated Systems Laboratory, ETH Zurich, Zurich, 8092 Switzerland (e-mail: cossettini.andrea@iis.ee.ethz.ch).

A. Ö. is with the Institute for Biomedical Engineering and Institute of Pharmacology and Toxicology, University of Zurich and ETH Zurich, Zurich, 8092 Switzerland (e-mail: oezbek@ethz.ch).

S. V. is with the Integrated Systems Laboratory, ETH Zurich, Zurich, 8092 Switzerland (e-mail: vsergei@iis.ee.ethz.ch).

P. H. is with the Integrated Systems Laboratory, ETH Zurich, Zurich, 8092 Switzerland (e-mail: phager@iis.ee.ethz.ch).

X. L. D. B. is with the Institute for Biomedical Engineering and Institute of Pharmacology and Toxicology, University of Zurich and ETH Zurich, Zurich, 8092 Switzerland (e-mail: xl.deanben@uzh.ch).

L. B. is with the Department of Electrical, Electronic, and Information Engineering, University of Bologna and the Integrated Systems Laboratory, ETH Zurich, Zurich, 8092 Switzerland (e-mail: Ibenini@iis.ee.ethz.ch).

D. R. is with the Institute for Biomedical Engineering and Institute of Pharmacology and Toxicology, University of Zurich and ETH Zurich, Zurich, 8092 Switzerland (e-mail: daniel.razansky@uzh.ch). 


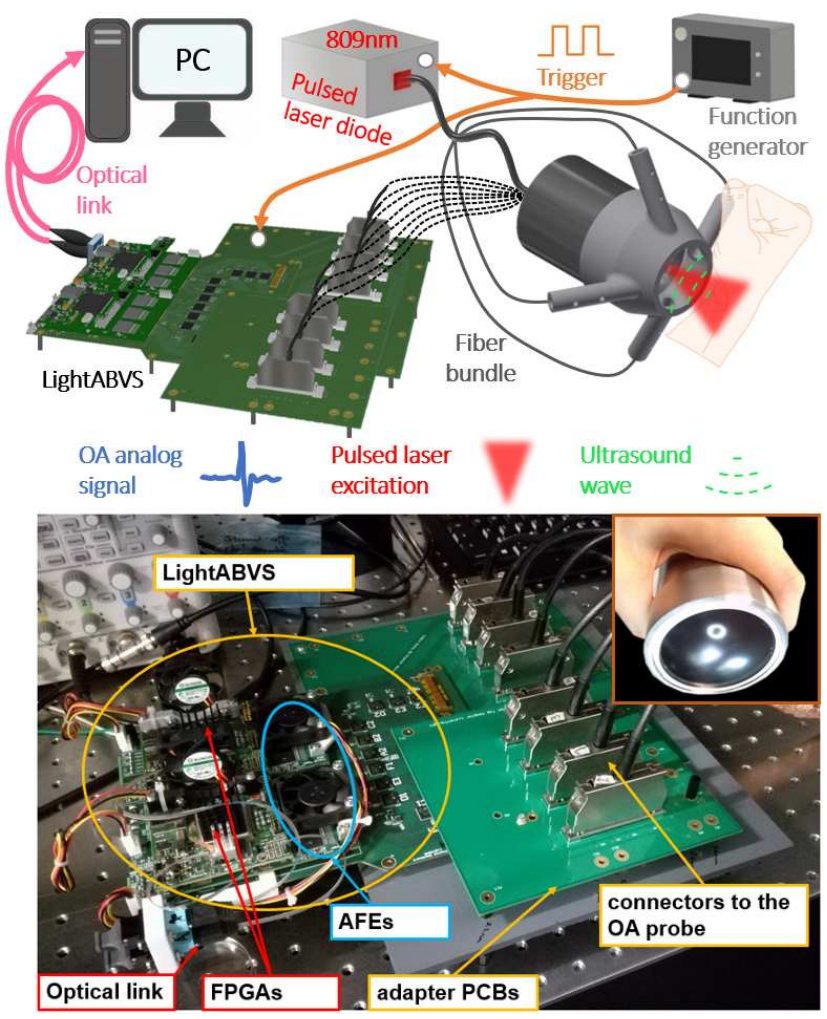

Fig. 1. Sketch of the main components of the handheld LightSpeed system (top). A pulsed laser diode source operating at $809 \mathrm{~nm}$ wavelength is coupled into a fiber bundle having 5 output arms for uniform illumination of the tissue surface. Absorption of the laser pulses results in tiny US waves that are captured by the array and sampled with the digital acquisition electronics (DAQ). An optical link connection to a host-PC enables fast data transfer for further processing. (Bottom). Photograph of the LightSpeed testbed with the handheld spherical array transducer probe shown in the inset. The bright spots in the photograph of the spherical transducer array occur due to glare. The custom DAQ system is connected to the transducer array via an adapter board. Image adapted from [27].

rate could only be increased by reducing the number of channels recorded for each laser pulse, i.e., by employing spatial subsampling of the tomographic data [22], [23]. These approaches are still afflicted with long reconstruction times [24] and reduced image quality. In addition, efficient compression can only be achieved if the imaged object manifests sufficient sparsity in some domain [25].

In this work, we introduce LightSpeed: a compact high-speed 3D OAT imager, based on a novel software-defined US digital acquisition architecture and a low-cost pulsed laser diode source [26], [27] to enable high-frame-rate OAT acquisitions. Imaging performance of the system is first validated with tissue mimicking phantoms and subsequently demonstrated by ultrafast $(500 \mathrm{~Hz}) 3 \mathrm{D}$ human angiography using a rapidly moving handheld probe.

\section{Materials And Methods}

\section{A. The LightSpeed System Design}

\section{A.1. Optical Illumination}

The LightSpeed imaging set-up developed in this work is illustrated in Fig. 1. Optoacoustic (OA) responses are generated by a pulsed laser diode (Lumibird SA, Lannion, France) that provides short ( $40 \mathrm{~ns}$ duration) laser pulses at $809 \mathrm{~nm}$ wavelength with a pulse repetition frequency (PRF) of up to several kHz. The per-pulse energy of the laser was set to its maximum value of $1.1 \mathrm{~mJ}$ for all experiments. The pulsed laser diode was powered by a 24 V DC supply (Voltcraft, LSP-1403) and externally triggered with a function generator (RIGOL, DG1022A). Its form-factor $(9 \mathrm{~cm} \times 5.6 \mathrm{~cm} \times 3.4 \mathrm{~cm})$ is much smaller compared to that of optical parametric oscillator (OPO)-based laser sources commonly employed for OAT applications [28], [29]. The light beam was guided through a custom-made silica fused-end fiber bundle consisting of 2415 individual fibers equally distributed between 5 output arms (CeramOptics GmbH, Bonn, Germany). One of the outputs was inserted into the central aperture of the spherical array transducer probe (Fig. 1, top). The four remaining bundle arms were equally spaced and fixed around the probe by means of a $3 \mathrm{D}$ printed holder. To ensure uniform illumination at the tissue surface, the angle and the distance between the fiber outputs of the 3D printed holder were carefully adjusted by taking into account the actual distance between the imaged object and transducer surface. This illumination design allowed to deliver energies of $\sim 0.65 \mathrm{~mJ}$ per-pulse to the tissue surface, distributed over an extended area of $\sim 5 \mathrm{~cm}^{2}$ corresponding to an energy density of approximately $0.13 \mathrm{~mJ} / \mathrm{cm}^{2}$. Under these conditions, the incident energy was kept well below the ANSI laser safety criteria for the human skin exposure $\left(\sim 40 \mathrm{~mJ} / \mathrm{cm}^{2}\right.$ at the $809 \mathrm{~nm}$ wavelength) [30]. Furthermore, for the maximum PRF of $500 \mathrm{~Hz}$ used in the current study, the average light intensity levels delivered to the skin surface similarly conformed to the safety standard of $400 \mathrm{~mW} / \mathrm{cm}^{2}$ at this wavelength.

\section{A.2. Optoacoustic Signal Acquisition}

The LightSpeed system accommodates a spherical matrix detection array consisting of 256 individual piezocomposite elements (Imasonic SaS, Voray, France), which collects the signals generated in the region of interest (ROI). The array features a central frequency of $4 \mathrm{MHz}$ with $100 \%$ detection bandwidth and covers a 90 degrees angle $(0.59 \pi$ solid angle) [31].

The US signal readout, processing and transmission in LightSpeed is based on the previously reported softwaredefined optical-link-based LightABVS platform for classical pulse-echo US imaging applications featuring 192 channels [26]. It has compact dimensions of $18 \mathrm{~cm} \times 22.6 \mathrm{~cm} \times 10 \mathrm{~cm}$ and is based on inexpensive off-the-shelf components with an overall cost of $\sim 7.400$ EUR and $\sim 2.000$ EUR for the PCBs design and fabrication). For pulse-echo US applications, highvoltage pulsers are required for generating the excitation signal: for this, LightABVS is equipped with 12x STHV1600 pulsers (STMicroelectronics, Geneva, Switzerland), enabling 192 channel operation. Active transmission is not required for the OAT imaging application that is done is a pure detection mode, thus the pulser chips are not operated with the internal transmit/receive switches locked on the receive path. The 


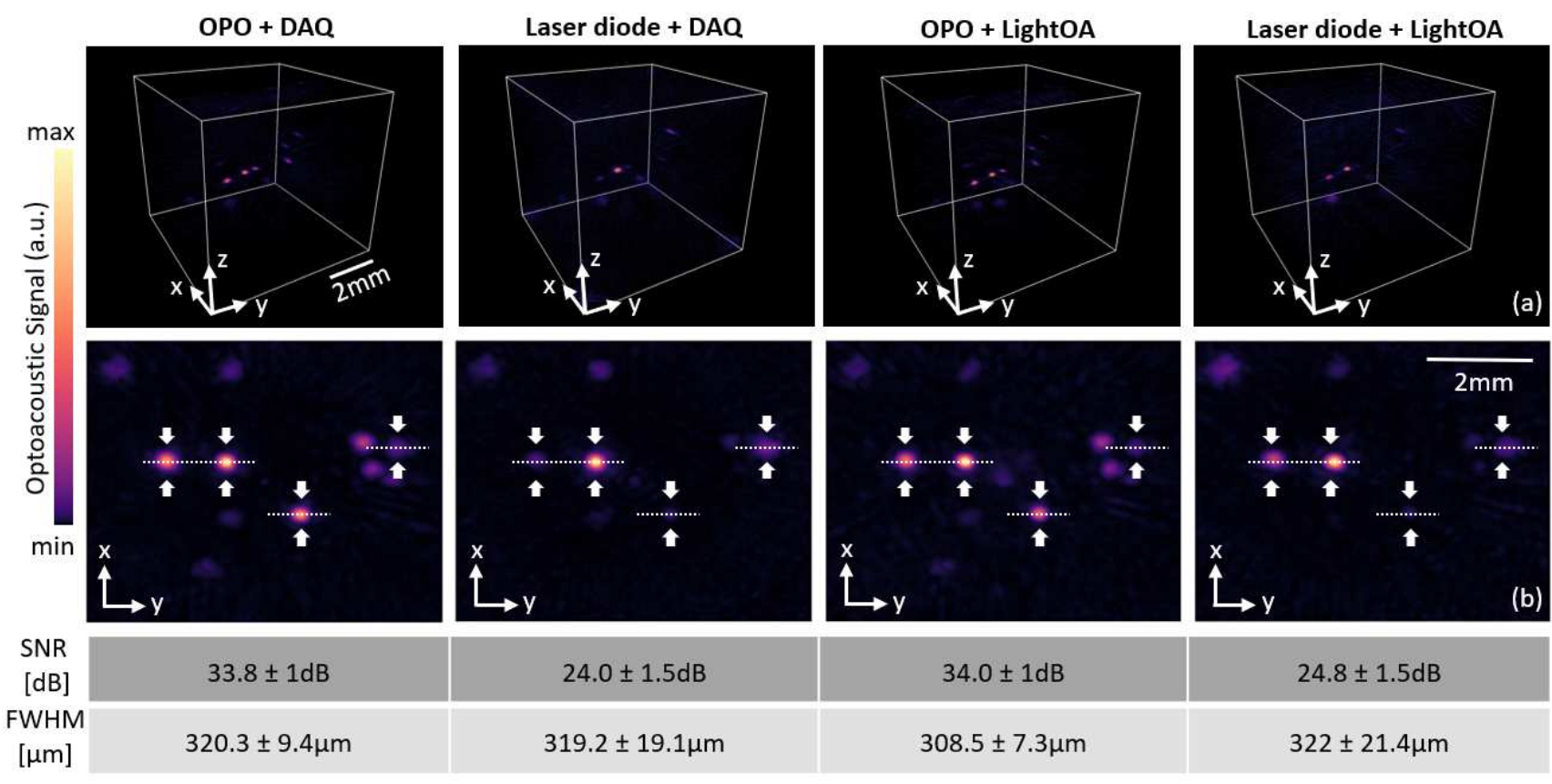

Fig. 2. Performance comparison between the developed compact LightSpeed imaging platform and a conventional OAT system featuring bulky Q-switched laser source and Ethernet-based digital acquisition (DAQ) interface. (a) Three-dimensional views of the reconstructed volumetric OAT images for 100- $\mu \mathrm{m}$ microspheres embedded in an agar phantom. Volumetric renderings are shown for four measurement configurations: Q-switched laser with conventional DAQ (first column), laser diode with conventional DAQ (second column), Q-switched laser with LightSpeed acquisition interface (third column) and laser diode with LightSpeed interface (fourth column). (b) Maximum intensity projections (MIPs) of the volumetric reconstructions along the axial (z) direction. The table reports SNR values (time-average) for the different configurations along with the full-width half maximum (FWHM) of the reconstructed microspheres along the dashed profiles shown in (b).

detected signals are amplified and digitized by means of six integrated analog front-end (AFE) modules from Texas Instruments (AFE58JD32), which guarantee a power efficiency of $42 \mathrm{~mW} /$ channel, typically unavailable in benchtop DAQ systems. The AFE operates at programmable sampling frequencies (typical value: $f_{s}=40 \mathrm{MHz}$ with 12-bit resolution). The digitized signals are fed to a field programmable gate array (FPGA) via JESD204B data converter serial interfaces.

The system is modular and is based on two Xilinx Kintex Ultrascale+ FPGAs (XCKU5P), devoted to controlling the overall system (e.g. managing the AFE configuration), aggregating the data coming from the AFEs (i.e. three AFE modules with 96 US channels per FPGA), and sending them to the host-PC for further processing. The communication between the FPGAs and the host-PC is based on a $100 \mathrm{G}$ Ethernet connection employing two Samtec 100G FireFly modules, and the data are transmitted as User Datagram Protocol (UDP) packets. The system is software-defined: arbitrary modes of operation can be implemented simply by adapting the control software and configuring the on-board FPGAs. Compared to other DAQ systems, this feature effectively enables to perform in-probe processing. Furthermore, it is effectively an open platform readily providing access to the raw data. LightSpeed supports highframe-rate modes of operation, currently operating at up to 500 $\mathrm{Hz}$ repetition rate for parallel acquisition from all its 192 channels. Even without aggressive power management, the system consumes only $37 \mathrm{~W}$ (192 mW/channel).
Two adapter printed circuit boards (PCBs) were designed to connect 192 selected channels of the matrix array transducer to the analog front-end (Fig. 1, bottom). The remaining 64 channels of the probe were terminated to ground with $50 \Omega$ resistors. For the imaging experiments, external triggering of the FPGAs was provided by the same function generator (RIGOL, DG1022A) that also triggers the pulsed laser diode (Fig. 1, top). For rendering each image frame, Ns $=1000$ samples are captured for each channel, corresponding to a $37.5 \mathrm{~mm}$-sized imaged region when assuming $1500 \mathrm{~m} / \mathrm{s}$ speedof-sound in soft tissues.

For performance comparison, we also acquired OAT image data with a benchtop Ethernet-based DAQ system $(\sim 48 \mathrm{~cm} \times$ $48 \mathrm{~cm} \times 20 \mathrm{~cm}$ dimensions) consisting of 512 parallel analogto-digital converters arranged in 16 acquisition cards (Falkenstein Mikrosysteme GmbH, Taufkirchen, Germany) previously reported for OA imaging applications [31], [32]. The DAQ consumes on average $370 \mathrm{~W}(723 \mathrm{~mW} /$ channel $)$ of electric power.

\section{B. Experimental Measurements}

\section{B.1. Phantom Measurements}

To validate performance of the LightSpeed system, we first measured the OA signals generated from $100-\mu \mathrm{m}$ lightabsorbing black paramagnetic polyethylene microspheres (Cospheric LLC, California, USA) embedded in an agar phantom. Four different measurement configurations were evaluated consisting of two different light sources and two 

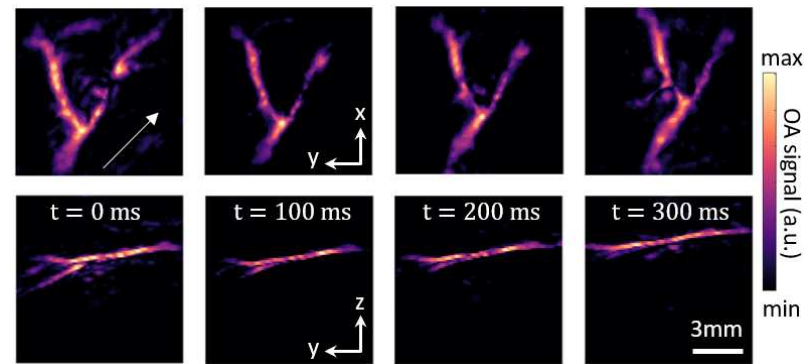

Fig. 3. Non-invasive volumetric OAT imaging of human vasculature. Maximum intensity projection OA images (normalized to the maximum) along $z$ (top panel) and $x$ (bottom panel) directions, taken from a healthy human volunteer (wrist region) using the LightSpeed system. The white arrow in the top-left panel indicates the direction of the handheld probe movement.

different digitization interfaces. Specifically, we compared the use of a tunable Q-switched laser source (InnoLas Laser GmbH, Krailling, Germany) emitting $<10$ ns duration pulses with the above described pulsed laser diode source. The per-pulse energy of the Q-switched laser source was set to $40 \mathrm{~mJ}$ and the measured energy at the output of the fiber bundle was $20 \mathrm{~mJ}$, corresponding to an energy density of approximately $4 \mathrm{~mJ} / \mathrm{cm}^{2}$ at the tissue surface. In turn, the conventional Ethernet-based DAQ was compared to the LightSpeed optical link-based interface. In all four measurement configurations, the spherical matrix array probe was filled with agar to ensure the acoustic coupling and fixed vertically on the optical table. An agar phantom containing light absorbing microspheres was placed on top of the vertically-fixed spherical array and aligned such that the microspheres were located approximately at the geometrical center of the sphere. Illumination wavelength of $809 \mathrm{~nm}$ was used for both light sources.

\section{B.2. In-vivo Measurements}

The general feasibility of performing deep tissue angiography with the handheld LightSpeed system was tested by imaging the wrist region of a healthy volunteer: we moved the transducer along an arbitrary path on the skin in free-hand mode. The speed of the ultrasound array motion was not directly controlled but estimated in post processing by calculating inter-frame displacements. Volumetric images of human vasculature were captured by moving the handheld probe along the region of interest (ROI). For the in vivo measurements, acoustic coupling was ensured by immersing the US probe and the arm of the volunteer in a water tank. Note that the $809 \mathrm{~nm}$ excitation wavelength of the laser diode is close to the isosbestic point of hemoglobin [33]. The pulse repetition rate was varied between 10 to $500 \mathrm{~Hz}$ for the dynamic data acquisition in the handheld mode. An extended area spanning $30 \mathrm{~mm}^{2}$ along the wrist region was scanned by moving the probe around the ROI.

\section{Image Reconstruction}

All volumetric OAT data were reconstructed using a Radontransform-based inversion algorithm [34], which involves deconvolution, band-pass filtering, and spherical backprojection steps. Image visualization and analysis were performed in Matlab (version 9.5, R2018b; MathWorks, Natick, Mass) and Amira (Zuse Institute, Berlin, Germany). The individual reconstructed volume is $10 \times 10 \times 10 \mathrm{~mm}^{3}$ in size and has $100 \times 100 \times 100$ voxels resolution.

\section{Image Filtering}

Due to the relatively low output energy of the laser diode, the impact of noise on the reconstructed OAT images was relatively high. In order to improve image quality, the reconstructed volumetric OAT images were first median-filtered with a window size of $3 \times 3 \times 3$ voxels. The median-filtered volumetric OAT data were then normalized with a threshold set to $0.3 \%$ of the brightest voxel to improve contrast.

\section{E. Image Compounding}

The employed spherical matrix array probe is characterized by a field-of-view (FOV) restricted to about $1 \mathrm{~cm}^{3}$ [35]. This limitation can be overcome by compounding multiple reconstructed volumes acquired by handheld motion [36], which further enables accurate estimation of the motion trajectory. The 3D spatial compounding algorithm adopted here is based on the cross-correlation of the Fourier spectra of consecutive frames and selection of the rotation angle and relative shift that attains the best fit. In our angiographic experiments, a total of 66 partially overlapping frames were compounded by means of an image-based Fourier domain motion estimation method [36] to render the anatomical structures on a larger spatial scale. Note that image

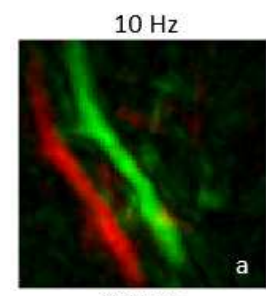

$100 \mathrm{~Hz}$

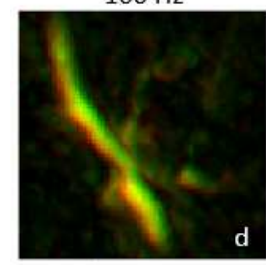

d
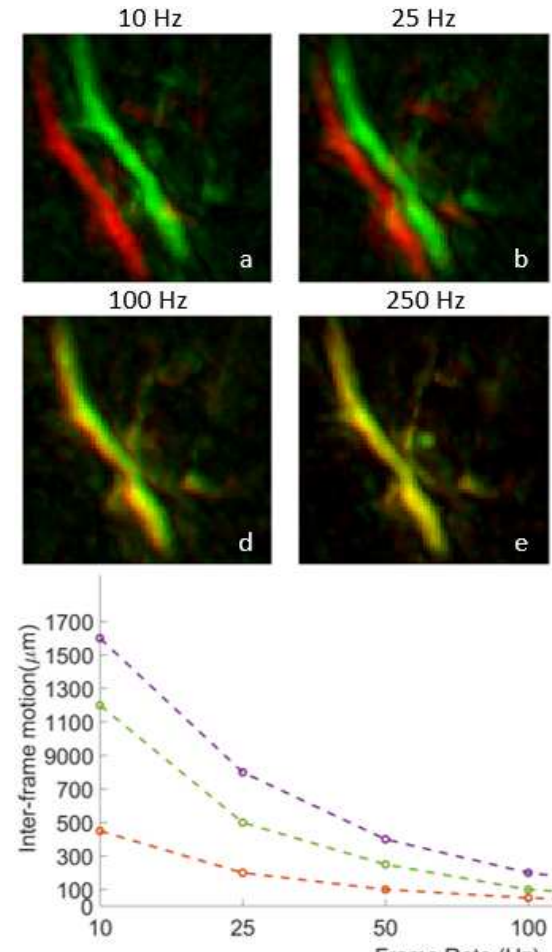

$250 \mathrm{~Hz}$
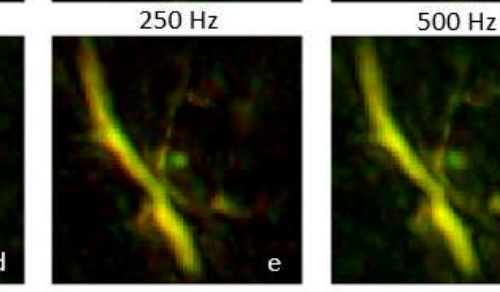
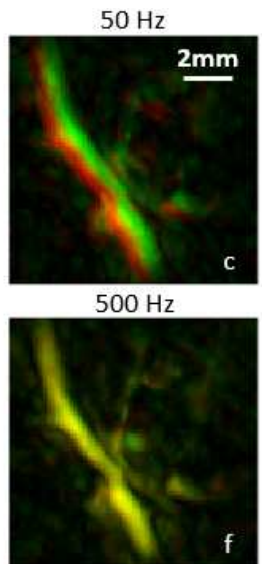

-a $16 \mathrm{~mm} / \mathrm{s} \quad \mathrm{g}$

$-\infty 12 \mathrm{~mm} / \mathrm{s}$

$-\infty .5 \mathrm{~mm} / \mathrm{s}$

Fig. 4. Inter-frame motion and assessment of the spatio-temporal resolution performance of the LightSpeed system. (a-f) Volumetric images recorded with laser pulse repetition rate ranging between 10 to $500 \mathrm{~Hz}$ - maximum intensity projections along the axial (z) dimension are shown. The scanning speed is $16 \mathrm{~mm} / \mathrm{s}$. Two consecutive frames are superimposed in red and green. (g) Average inter-frame motion $(\mu \mathrm{m})$ between the vascular features plotted against the frame rate employed for three different probe scanning speeds: fast $(16 \mathrm{~mm} / \mathrm{s})$, moderate $(12 \mathrm{~mm} / \mathrm{s})$, and slow (4.5 mm/s). 


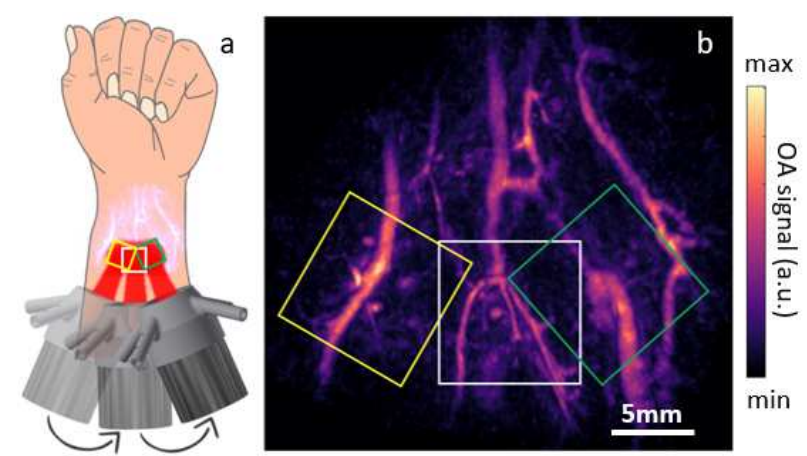

Fig. 5. Image rendering by compounding a volumetric image sequence acquired via freehand scanning of the OAT probe. (a) Diagram illustrating the handheld scanning procedure, where the probe is scanned around the wrist area. (b) Large FOV rendered via image-based Fourier domain motion estimation and compounding 66 consecutive image volumes [36]. Axial projection of volumetric image is shown.

compounding effectively results in signal averaging across overlapping regions between the adjucent frames.

\section{RESULTS}

Comparison between the volumetric OAT images rendered from the microsphere phantom by means of the four tested illumination-detection configurations is presented in Fig. 2. It is shown that the recorded number of 192 tomographic projections (channels) is sufficient for accurately rendering the spheres without resolution degradation. Overall, the images generated with the conventional DAQ system are also consistent with the results obtained with LightSpeed, attaining similar signal-to-noise ratio (SNR) and spatial full-width halfmaximum (FWHM) values for the reconstructed microspheres (table under Fig. 2b). The presented SNR values were calculated based on the same (most visible) microsphere for all four illumination-detection configurations. Note that differences in the illumination profiles from both lasers affect the signals of each sphere. The SNR values are averaged in time (computed over multiple subsequent frames) and presented with the corresponding standard deviation values, i.e. $\pm 1.5 \mathrm{~dB}$ and $\pm 1 \mathrm{~dB}$ for the laser diode and for the OPO laser, respectively. The FWHM values were calculated by extracting the optoacoustic signal profiles along the dashed lines shown in Fig. $2 b$ and subsequent fitting to a one-dimensional Gaussian function. The average FWHM values of four microspheres are presented. As expected, SNR values were higher when the Qswitched laser was used due to its higher per-pulse energy output as compared to the laser diode.

Following the phantom validation experiments, visualization of the human vasculature was performed in a freehand mode. Fig. 3 shows four consecutive unaveraged volumetric OA images of blood vessels in the wrist of a healthy volunteer acquired at $10 \mathrm{~Hz}$ PRF of the laser. Vasculature of varying size was clearly resolved up to a depth of $5 \mathrm{~mm}$ by means of the LightSpeed system.

To facilitate a reliable image compounding and to further demonstrate the handheld imaging capability, the imaging was performed with the scanning speed increased from $4.5 \mathrm{~mm} / \mathrm{s}$ (slow) to $12 \mathrm{~mm} / \mathrm{s}$ (moderate), to $16 \mathrm{~mm} / \mathrm{s}$ (fast). The interframe motion was calculated by cross-correlation of consecutive image frames (2D projections along the axial $\mathrm{z}$ dimension). To estimate the effective spatio-temporal resolution of the measurements, we estimated the overlap between two subsequent image frames acquired at different frame rates (Fig. 4). The scanning speeds of the ultrasound array were then calculated by dividing the inter-frame displacement by the time between two consecutive image frames. While substantial displacement of the blood vessels between the two frames can be observed at $10 \mathrm{~Hz}$ acquisition rate (Fig. 4a), the frames become perfectly aligned when the frame rate is increased to $500 \mathrm{~Hz}$ (Fig. 4f). We further calculated the spatial shift between two consecutive frames as a function of frame rate for the three scanning speeds (Fig. $4 \mathrm{~g}$ ). In the fast scanning mode, the inter-frame motion amounts to $1.6 \mathrm{~cm}$ at $10 \mathrm{~Hz}$ and $50 \mu \mathrm{m}$ at $500 \mathrm{~Hz}$, i.e. below spatial resolution of the imaging system. In the slow scanning mode, the inter-frame motion is only $450 \mu \mathrm{m}$ at $10 \mathrm{~Hz}$, dropping to unrecognizable values at 500 Hz. Finally, results of the spatial compounding procedure are shown in Fig. 5, further corroborating the large-scale handheld imaging capacity of the developed LightSpeed platform.

\section{Discussion And Conclusion}

This work demonstrates the feasibility of high-frame-rate volumetric OAT imaging with a compact, low-cost, and configurable LightSpeed system. It features a novel custommade optical link-based parallel signal acquisition and a fibercoupled laser diode illumination configuration. LightSpeed thus paves the way toward realizing affordable handheld OAT scanners particularly suitable for bedside clinical imaging applications [37].

Imaging performance of the new system was found similar to what can be achieved with standard benchtop DAQ systems used in OAT [38], [39]. However, OA signals are proportional to the per-pulse laser energy, thus resulting in a relatively low SNR for the typical $\sim 1 \mathrm{~mJ}$ optical energies provided by laser diodes [28], in contrast with the high energy OPO-based lasers sources. The optoacoustic signals and their SNR are further affected by pulse-to-pulse laser energy variations as well as instability of the illumination beam profile. The alignment between the sensor head and the excitation light beam is of particular importantance for achieving optimal performance in terms of image contrast, SNR, dynamic range and effective penetration depth. Other design considerations include the light beam pattern and its dimension on the tissue surface - affecting the dynamic range and penetration depth - as well as the depth of the acoustic focus of the array relative to the skin, which affects the achievable SNR. In our experiments, the achieved SNR was adequate for clearly resolving vascular structures at depths of up to $5 \mathrm{~mm}$. The lower SNR with respect to that achieved with common Q-switched lasers can be further compensated by averaging multiple frames acquired at higher PRFs, which was shown to be feasible with the developed platform. Note however that the pulse width of the light source also has an influence on bandwidth of the generated OA signals 
[40], which may limit the achievable spatial resolution when employing laser diode sources for the excitation.

Real-time preview of optoacoustic images during hand-held scans is of particular importance. In this respect, the analytical back-projection inverse algorithms [34], [41] or other coherence-based beamforming methods [42], [43], [44] are suitable for real-time image rendering, in particular when implemented in a graphics processing unit (GPU) [45], [46]. Image quality and quantification can be further improved by accounting for common experimental factors [47], [48], [49] or by employing more accurate model-based reconstruction algorithms [50], albeit at the expense of a slower computational speed.

Imaging at a high frame rate is required for mitigating interframe motion when performing fast handheld scans. This is of particular importance for multi-spectral optoacoustic tomography (MSOT) applications where pulses at multiple wavelengths are launched in an alternate fashion to resolve spectroscopically the distribution of distinct tissue chromophores [51], [52]. In this case, even slight shifts between features in consecutive frames acquired at different wavelengths may significantly hamper the spectral differentiation capacity [53]. Large displacements between consecutive frames may also hinder efficient compounding of image sequences thus results in inaccurate translation estimations [36], especially when employing lower laser pulse repetition frequencies in combination with fast motion of the ultrasound array. On the other hand, laser safety remains an important consideration when imaging at high frame rates. Both per-pulse energy density and average light intensity on the tissue surface must be kept below the laser exposure limits in order to ensure safe operation [30]. For our experiments the pulse repetition frequency has not exceeded $500 \mathrm{~Hz}$, for which the safety limits were fulfilled while the displacement between frames was kept under system's spatial resolution even for relatively fast handheld motion of $16 \mathrm{~mm} / \mathrm{s}$.

A large room exists for boosting the performance of the LightSpeed platform. The available bandwidth of the fiber optical link is generally capable of accommodating OA signal acquisition at significantly higher PRFs, provided the software on the host computer is configured to efficiently handle the received data. In addition, vast efforts have been recently directed towards the integration of OAT and reflection/transmission US imaging capacities in the same scanner [54], [55] whereas fast signal acquisition is essential for enabling ultrafast US imaging [56], [57].

In conclusion, the newly developed platform demonstrates the feasibility of a new generation of compact, affordable, and high-performance handheld OAT scanners, which is expected to facilitate clinical translation of this imaging technology. The capability to acquire OAT images at volumetric rates of hundreds to thousands of $\mathrm{Hz}$ is also poised to play an important role in research studies involving visualization of rapid biological dynamics, such as neural activity, cardiac function and free animal behavior.

\section{ACKNOWLEDGMENTS}

The authors thank A. Blanco Fontao and H. Gisler (ETH Zürich) for technical support.

\section{REFERENCES}

[1] Ç. Özsoy, M. Floryan, X. L. Deán-Ben and D. Razansky, "Endocardial irrigated catheter for volumetric optoacoustic mapping of radio-frequency ablation lesion progression”, Opt. Lett., vol. 44, no. 23, pp. 5808-5811, 2019.

[2] J. Lv et al., "Hemispherical photoacoustic imaging of myocardial infarction: in vivo detection and monitoring", Eur. Radiol., vol. 28, no. 5, pp. 2176-2183, 2018.

[3] V. Periyasamy, Ç. Özsoy, M. Reiss, X. L. Deán-Ben and D. Razansky, "In vivo optoacoustic monitoring of percutaneous laser ablation of tumors in a murine breast cancer model”, Opt. Lett., vol. 45, no. 7, pp. 2006-2009, 2020.

[4] S. P. Johnson, O. Ogunlade, M. F. Lythgoe, P. Beard and R. B. Pedley, "Longitudinal photoacoustic imaging of the pharmacodynamic effect of vascular targeted therapy on tumors", Clin. Cancer Res., vol. 25, no. 24, pp. 7436-7447, 2019.

[5] R. Cao, J. Li, C. Zhang, Z. Zuo and S. Hu, "Photoacoustic microscopy of obesity-induced cerebrovascular alterations", Neuroimage, vol. 188, pp. 369-379, 2019.

[6] L. Li, J. Yao and L. V. Wang, "Photoacoustic Tomography of Neural Systems," in Neural Engineering: Springer, 2020, pp. 349-378.

[7] S. V. Ovsepian, I. Olefir, G. Westmeyer, D. Razansky and V. Ntziachristos, "Pushing the boundaries of neuroimaging with optoacoustics", Neuron, vol. 96, no. 5, pp. 966-988, 2017.

[8] A. Ron, X. L. Deán-Ben, S. Gottschalk and D. Razansky, "Volumetric optoacoustic imaging unveils high-resolution patterns of acute and cyclic hypoxia in a murine model of breast cancer", Cancer Res., vol. 79, no. 18, pp. 4767-4775, 2019.

[9] I. Ivankovic et al., "Volumetric optoacoustic tomography enables noninvasive in vivo characterization of impaired heart function in hypoxic conditions", Sci. Rep., vol. 9, no. 1, pp. 1-8, 2019.

[10] A. P. Regensburger et al., "Detection of collagens by multispectral optoacoustic tomography as an imaging biomarker for Duchenne muscular dystrophy", Nat. Med., vol. 25, no. 12, pp. 1905-1915, 2019.

[11] G. Diot et al., "Multispectral optoacoustic tomography (MSOT) of human breast cancer", Clin. Cancer Res., vol. 23, no. 22, pp. 6912-6922, 2017.

[12] F. Knieling et al., "Multispectral Optoacoustic Tomography for Assessment of Crohn's Disease Activity", N. Engl. J. Med., vol. 376, no. 13, pp. 1292-1294, 2017.

[13] K. K. Shung, "Diagnostic ultrasound: Past, present, and future", J. Med. Biol. Eng., vol. 31, no. 6, pp. 371-374, 2011.

[14] A. Eklund, P. Dufort, D. Forsberg and S. M. LaConte, "Medical image processing on the GPU-Past, present and future", Med. Image Anal., vol. 17, no. 8, pp. 1073-1094, 2013.

[15] M. I. Fuller, T. N. Blalock, J. A. Hossack and W. F. Walker, "A portable, low-cost, highly integrated, 3D medical ultrasound system" in IEEE IUS, 2003, vol. 1, pp. 38-41.

[16] L. Ding, X. L. Deán-Ben, and D. Razansky, "Real-time model-based inversion in cross-sectional optoacoustic tomography", IEEE Trans. Med. Imaging, vol. 35, no.8, pp. 1883-1891, 2016.

[17] A. Buehler, M. Kacprowicz, A. Taruttis, and V. Ntziachristos, "Real-time handheld multispectral optoacoustic imaging", Opt. Lett., vol. 38, no. 9, pp. 1404-1406, 2013.

[18] X. L. Deán-Ben, E. Bay, and D. Razansky (2015, March). Threedimensional multispectral hand-held optoacoustic imaging with microsecond-level delayed laser pulses. In Photons Plus Ultrasound: Imaging and Sensing 2015 (Vol. 9323, p. 93231N). International Society for Optics and Photonics.

[19] S. Gottschalk, T. F. Fehm, X .L. Deán-Ben, V. Tsytsarev, and D. Razansky, "Correlation between volumetric oxygenation responses and electrophysiology identifies deep thalamocortical activity during epileptic seizures", Neurophotonics, vol. 4, no. 1, pp. 011007, 2016.

[20] H. C. A. Lin et. al., "Characterization of cardiac dynamics in an acute myocardial infarction model by four-dimensional optoacoustic and magnetic resonance imaging", Theranostics, vol. 7, no. 18, pp. 44704479, 2017.

[21] S. Gottschalk, T. F. Fehm, X. L. Deán-Ben, and D. Razansky, "Noninvasive real-time visualization of multiple cerebral hemodynamic 
parameters in whole mouse brains using five-dimensional optoacoustic tomography”, J. Cereb. Blood Flow Metab., vol. 35, no. 4, pp. 531-535, 2015.

[22] C. G. Graff, and E. Y. Sidky, "Compressive sensing in medical imaging”, Appl. Opt., vol. 54, no. 8, pp. C23-C44, 2015.

[23] Z. Guo, C. Li, L. Song, and L. V. Wang, "Compressed sensing in photoacoustic tomography in vivo", J. Biomed. Opt., vol. 15, no. 2, pp. $021311,2010$.

[24] A. Özbek, X. L. Deán-Ben, and D. Razansky, "Optoacoustic imaging at kilohertz volumetric frame rates", Optica, vol. 5, no. 7, pp. 857-863, 2018.

[25] D. L. Donoho, "Compressed sensing”, IEEE Trans. Inf. Theory, vol. 52, no. 4, pp. 1289-1306, 2006.

[26] P. A. Hager, F. Kuhn Jush, M. Biele, P. M. Düppenbecker, O. Schmidt, and L. Benini, "LightABVS: A Digital Ultrasound Transducer for MultiModality Automated Breast Volume Scanning”, in IEEE IUS, 2019.

[27] Ç. Özsoy et al., "Towards a compact, high-speed optical link-based 3D optoacoustic imager," in Proc. IEEE Sensors, 2020.

[28] M. K. A. Singh, and W. Xia, "Portable and Affordable Light SourceBased Photoacoustic Tomography", Sensors, vol. 20, no. 21, pp. 6173, 2020 .

[29] H. Zhong, T. Duan, H. Lan, M. Zhou, and F. Gao, "Review of low-cost photoacoustic sensing and imaging based on laser diode and lightemitting diode", Sensors, vol. 18, no. 7, pp. 2264, 2018.

[30] Ansi, Z. (2000). 136.1 „American National Standard for Safe Use of Lasers. Laser Institute of America, Orlando.

[31] X. L. Deán-Ben, A. Ozbek and D. Razansky, "Volumetric real-time tracking of peripheral human vasculature with GPU-accelerated threedimensional optoacoustic tomography”, IEEE Trans. Med. Imaging, vol. 32, no. 11, pp. 2050-2055, 2013.

[32] E. Merčep, X. L. Deán-Ben and D. Razansky, "Combined pulse-echo ultrasound and multispectral optoacoustic tomography with a multisegment detector array”, IEEE Trans. Med. Imaging, vol. 36, no. 10, pp. 2129-2137, 2017.

[33] X. Deán-Ben, S. Gottschalk, B. Mc Larney, S. Shoham and D. Razansky, "Advanced optoacoustic methods for multiscale imaging of in vivo dynamics”, Chem. Soc. Rev., vol. 46, no. 8, pp. 2158-2198, 2017.

[34] A. Ozbek, X. Deán-Ben and D. Razansky (2013, May). Realtime parallel back-projection algorithm for three-dimensional optoacoustic imaging devices. In European conference on biomedical optics (p. 88000I). Optical Society of America.

[35] X. L. Deán-Ben and D. Razansky, "Portable spherical array probe for volumetric real-time optoacoustic imaging at centimeter-scale depths", Opt. Express, vol. 21, no. 23, pp. 28062-28071, 2013.

[36] N. Knauer, X. L. Deán-Ben and D. Razansky, "Spatial Compounding of Volumetric Data Enables Freehand Optoacoustic Angiography of LargeScale Vascular Networks", IEEE Trans. Med. Imaging, vol. 39, no. 4, pp. 1160-1169, 2019.

[37] V. Neuschmelting et al., "Performance of a multispectral optoacoustic tomography (MSOT) system equipped with 2D vs. 3D handheld probes for potential clinical translation", Photoacoustics, vol. 4, no. 1, pp. 1-10, 2016

[38] M. K. A. Singh, W. Steenbergen, and S. Manohar, "Handheld probebased dual mode ultrasound/photoacoustics for biomedical imaging", In Frontiers in Biophotonics for Translational Medicine: Springer, 2016, pp. 209-247.

[39] Y. Bai, B. Cong, X. Gong, L. Song, and C. Liu, "Compact and low-cost handheld quasibright-field linear-array probe design in photoacoustic computed tomography", J. Biomed. Opt., vol. 23, no. 12, pp. 1408-1422, 2018.

[40] K. Irisawa, T. Hirasawa, K. Hirota, K. Tsujita and M. Ishihara (2012, February). Influence of laser pulse width to the photoacoustic temporal waveform and the image resolution with a solid-state excitation laser. In Photons Plus Ultrasound: Imaging and Sensing 2012 (Vol. 8223, pp. 82232 W). International Society for Optics and Photonics.

[41] A. Buehler, X. L. Deán-Ben, J. Claussen, V. Ntziachristos, and D. Razansky, "Three-dimensional optoacoustic tomography at video rate", Opt. Express, vol. 20, no. 20, pp. 22712-22719, 2012.

[42] Y. -H. Wang, and P. -C. Li, "SNR-dependent coherence-based adaptive imaging for high-frame-rate ultrasonic and photoacoustic imaging", IEEE Trans. Ultrason. Ferroelectr. Freq. Control, vol. 61, no. 8, pp. 1419-1432, 2014.

[43] M. T. Graham, and M. A. L. Bell, "Photoacoustic spatial coherence theory and applications to coherence-based image contrast and resolution", IEEE
Trans. Ultrason. Ferroelectr. Freq. Control, vol. 67, no. 10, pp. 2069-2084, 2020.

[44] R. A. Mukaddim and T. Varghese, "Spatiotemporal Coherence Weighting for In Vivo Cardiac Photoacoustic Image Beamformation", IEEE Trans. Ultrason. Ferroelectr. Freq. Control, vol. 68, no. 3, pp. 586-598, 2021.

[45] K. Wang, C. Huang, Y. J. Kao, C. Y. Chou, A. A. Oraevsky and M. A. Anastasio, "Accelerating image reconstruction in three-dimensional optoacoustic tomography on graphics processing units", Med. Phys., vol. 40, no. 2, pp. 023301, 2013.

[46] E. Gonzalez, and M. A. L. Bell, "GPU implementation of photoacoustic short-lag spatial coherence imaging for improved image-guided interventions", J. Biomed. Opt., vol. 25, no. 7, pp. 077002, 2020.

[47] X. L. Deán-Ben, V. Ntziachristos, and D. Razansky, "Artefact reduction in optoacoustic tomographic imaging by estimating the distribution of acoustic scatterers," J. Biomed. Opt. vol. 17, no. 11, pp. 110504, 2012.

[48] A. Rosenthal, V. Ntziachristos, and D. Razansky, "Model-based optoacoustic inversion with arbitrary-shape detectors", Med. Phys., vol. 38, no. 7, pp. 4285-4295, 2011.

[49] A. Rosenthal, D. Razansky, and V. Ntziachristos, "Quantitative Optoacoustic Signal Extraction Using Sparse Signal Representation", IEEE Trans. Med. Imaging, vol. 28, no. 12, pp. 1997-2006, 2009.

[50] X. L. Deán-Ben, A. Buehler, V. Ntziachristos and D. Razansky, "Accurate model-based reconstruction algorithm for three-dimensional optoacoustic tomography”, IEEE Trans. Med. Imaging, vol. 31, no. 10, pp. 1922-1928, 2012.

[51] X. L. Deán-Ben, E. Bay, and D. Razansky, "Functional optoacoustic imaging of moving objects using microsecond-delay acquisition of multispectral three-dimensional tomographic data", Sci. Rep., vol. 4, pp. $1-6,2014$.

[52] A. Taruttis, and V. Ntziachristos, "Advances in real-time multispectral optoacoustic imaging and its applications", Nat. Photonics, vol. 9, no. 4, pp. 219-227, 2015.

[53] H. Li, I. Ivankovic, J. Li, D. Razansky and X. L. Deán-Ben, "Coregistration and Spatial Compounding of Optoacoustic Cardiac Images via Fourier Analysis of Four-Dimensional Data", Appl. Sci., vol. 10 , no. 18 , pp. 6254,2020

[54] E. Merčep, J. L. Herraiz, X. L. Deán-Ben and D. Razansky, "Transmission-reflection optoacoustic ultrasound (TROPUS) computed tomography of small animals", Light Sci. Appl., vol. 8, no. 1, pp. 1-12, 2019.

[55] M. Oeri, W. Bost, N. Sénégond, S. Tretbar and M. Fournelle, "Hybrid photoacoustic/ultrasound tomograph for real-time finger imaging", Ultrasound Med. Biol., vol. 43, no. 10, pp. 2200-2212, 2017.

[56] M. Tanter, and M. Fink, "Ultrafast imaging in biomedical ultrasound", IEEE Trans. Ultrason. Ferroelectr. Freq. Control, 61(1), 102-119, 2014.

[57] M. Cikes, L. Tong, G. R. Sutherland, and J. D'hooge, "Ultrafast cardiac ultrasound imaging: technical principles, applications, and clinical benefits”, JACC: Cardiovasc. Imaging, vol. 7, no. 8, pp. 812-823, 2014. 